\title{
Diagnostic delay is common among patients with hypophosphatasia: initial findings from a longitudinal, prospective, global registry
}

Wolfgang Högler ${ }^{1 \dagger}$, Craig Langman ${ }^{2 \dagger}$, Hugo Gomes da Silva ${ }^{3}$, Shona Fang ${ }^{3}$, Agnès Linglart ${ }^{4}$, Keiichi Ozono ${ }^{5}$, Anna Petryk ${ }^{3}$, Cheryl Rockman-Greenberg ${ }^{6}$, Lothar Seefried ${ }^{7}$ and Priya S. Kishnani ${ }^{8^{*+}}$

\begin{abstract}
Background: Hypophosphatasia (HPP) is a rare, systemic disease caused by mutation(s) within the ALPL gene encoding tissue-nonspecific alkaline phosphatase (ALP). HPP has a heterogeneous presentation, which coupled with its rarity, often leads to missed/delayed diagnosis and an incomplete understanding of its natural history. To better understand the epidemiology and clinical course of HPP, including timing of diagnosis after first reported manifestation, we present baseline data for patients enrolled in the Global HPP Registry.

Methods: Data were analyzed from patients with an HPP diagnosis confirmed by low serum ALP activity and/or an ALPL pathogenic variant, regardless of prior or current treatment, according to age at enrollment (children: $<18$ y; adult: $\geq 18$ y). All analyses were descriptive.

Results: Of 269 patients from 11 countries enrolled January 2015-September 2017, 121 (45.0\%) were children and 148 (55.0\%) were adults. The majority of children and adults were female (61.2 and $73.0 \%$, respectively) and white (57.7 and 90.0\%, respectively). Children had a median ( $\mathrm{min}, \mathrm{max}$ ) age at earliest reported HPP manifestation of 7.2 months $(-2.3 \mathrm{mo}, 16.0 \mathrm{y})$, which was $>12$ months before diagnosis at age 20.4 months $(-0.2 \mathrm{mo}, 16.0 \mathrm{y})$. In adults, the earliest reported manifestation occurred at a median (min, max) age of 37.6 years $(0.2 \mathrm{y}, 75.2 \mathrm{y})$, which preceded age at diagnosis ( 47.5 years [0.2 y, $75.2 \mathrm{y}]$ ) by $\sim 10$ years. Premature loss of deciduous teeth $(48.2 \%$, age $\geq 6 \mathrm{mo})$, bone deformity (32.5\%), and failure to thrive (26.7\%) were most commonly reported in the HPP-related disease history of children. Pain (74.5\%), orthopedic procedures and therapies (44.6\%), and recurrent and poorly healing fractures (36.5\%) were most commonly reported in the HPP-related disease history of adults.

Conclusions: The Global HPP Registry represents the largest observational study of patients with HPP, capturing real world data. This analysis shows that diagnostic delay is common, reflecting limited awareness of HPP, and that HPP is associated with systemic manifestations across all ages. Many patients diagnosed in adulthood had HPP manifestations in childhood, highlighting the importance of taking thorough medical histories to ensure timely diagnosis.
\end{abstract}

Trial registration: Clinicaltrials.gov: NCT02306720, December 2014; ENCePP.eu: EUPAS13526, May 2016 (retrospectively registered).

Keywords: Hypophosphatasia, Natural history, Rare diseases, Alkaline phosphatase, Asfotase alfa

\footnotetext{
* Correspondence: priya.kishnani@duke.edu

${ }^{\dagger}$ Wolfgang Högler, Craig Langman and Priya S. Kishnani contributed equally

as co-lead authors and senior author, respectively; the remaining authors are

listed alphabetically to reflect their equal contributions.

${ }^{8}$ Department of Pediatrics, Duke University Medical Center, 2301 Erwin Rd,

Durham, NC 27710, USA

Full list of author information is available at the end of the article
}

(c) The Author(s). 2019 Open Access This article is distributed under the terms of the Creative Commons Attribution 4.0 International License (http://creativecommons.org/licenses/by/4.0/), which permits unrestricted use, distribution, and reproduction in any medium, provided you give appropriate credit to the original author(s) and the source, provide a link to the Creative Commons license, and indicate if changes were made. The Creative Commons Public Domain Dedication waiver (http://creativecommons.org/publicdomain/zero/1.0/) applies to the data made available in this article, unless otherwise stated. 


\section{Background}

Hypophosphatasia (HPP) is a rare, inherited, systemic, metabolic disease caused by genetically determined low tissue-nonspecific alkaline phosphatase (TNSALP) activity [1-4]. Low TNSALP activity leads to the extracellular accumulation of TNSALP substrates (e.g., inorganic pyrophosphate [PPi], pyridoxal 5' -phosphate [PLP]) $[5,6]$, resulting in both bone mineralization defects and systemic complications $[3,7]$. Because of the rarity of HPP, the incidence and prevalence of this disease are difficult to estimate [3]. Commonly cited rates in the literature include an incidence of 1:100,000 in Toronto based on the local birth rate for Ontario, Canada [8], and a prevalence of 1:300,000 for perinatal/infantile HPP in France based on molecular diagnoses made from 2000 to 2009 [9]. Additional estimates include a 1:2500 prevalance of infantile HPP among the Mennonite population in Manitoba, Canada, based on live births [10], an incidence of 1:300,000-500,000 for fatal perinatal HPP in Japan [11], and a 1:900,000 prevalance of a founder mutation (c.1559delT) in Japan based on genetic analysis [12].

The clinical expression of HPP is heterogeneous, even among individuals with the same genotype or in the same family $[2,13]$. Certain signs, symptoms, or complications of HPP may be more common based on the patient's age $[2,3]$. The characteristic manifestations of HPP in infants may include failure to thrive, rickets-like chest deformity, pulmonary insufficiency, craniosynostosis, and vitamin B6-responsive seizures; in toddlers, young children, and adolescents, premature tooth loss, bone deformities and rachitic-changes in long bones, and delayed motor development manifest; and, in adults, musculoskeletal pain, chondrocalcinosis (pseudogout), recurrent fractures, poorly healing fractures, or pseudofractures are characteristic $[2,3,8]$. The natural history of HPP is poorly understood at this time, likely because of the rarity and wide heterogeneity of disease presentation. Further, real world data are lacking on the disease burden for patients with HPP and, in particular, on the impact of the disease on physical function and quality of life.

Until 2015, clinical management of HPP relied mainly on supportive measures that managed the symptoms of the disease (e.g., respiratory support, orthopedic intervention, pain relief medication) but not the underlying pathophysiology $[2,3,14,15]$. In 2015, asfotase alfa (Strensiq ${ }^{\oplus}$, Alexion Pharmaceuticals, Inc., Boston, MA, USA), a human recombinant TNSALP enzyme replacement therapy, was approved for the treatment of patients with HPP [16-18].

The Global HPP Registry was established to improve the understanding of the disease and its impact on patients with HPP. In addition, the Registry collects data on the effectiveness and safety and tolerability of treatment with asfotase alfa as part of a postmarketing commitment to monitor real world safety and use of asfotase alfa. Data on HPP history, clinical course, signs/ symptoms/complications, and burden of disease are collected from patients of all ages diagnosed with HPP, regardless of treatment status. This report presents baseline characteristics and medical history of patients enrolled in the Registry.

\section{Methods}

The Global HPP Registry is an observational, prospective, multinational study (NCT02306720; EUPAS13526), with medical history data collected based on patient or parent/ guardian recall. The Registry is sponsored by Alexion Pharmaceuticals, Inc. (Boston, MA, USA), and is overseen by a scientific advisory board comprising HPP clinical experts, including employees of Alexion Pharmaceuticals, Inc. The study protocol was approved by the institutional review board (or local equivalent) of participating study sites (see Acknowledgments section) and is being conducted in accordance with International Conference on Harmonisation Good Clinical Practice Guidelines and the Declaration of Helsinki. Before participating, all patients and/or their parent/legal guardians provided written informed consent and approval to release medical records.

\section{Patients}

Patients of all ages who have a confirmed diagnosis of HPP are eligible for participation in the HPP Registry, regardless of asfotase alfa treatment status. Patients who were deceased before enrollment are not included in the Registry. Patients who had previously participated in an Alexion-sponsored study of asfotase alfa are allowed to

Table 1 Number of sites and patients enrolled in the HPP Registry by age at enrollment

\begin{tabular}{|c|c|c|c|c|}
\hline \multirow[b]{2}{*}{ Country } & \multicolumn{4}{|c|}{ Patients Enrolled, ${ }^{a} \mathrm{n}$} \\
\hline & No. of Sites & $\begin{array}{l}\text { Children } \\
\text { (age < 18 y) }\end{array}$ & $\begin{array}{l}\text { Adults } \\
\text { (age } \geq 18 \mathrm{y} \text { ) }\end{array}$ & Total \\
\hline United States & 16 & 38 & 76 & 114 \\
\hline United Kingdom & 6 & 24 & 13 & 37 \\
\hline Japan & 23 & 28 & 2 & 30 \\
\hline Spain & 7 & 5 & 18 & 23 \\
\hline Canada & 1 & 8 & 13 & 21 \\
\hline France & 2 & 6 & 9 & 15 \\
\hline Australia & 2 & 10 & 1 & 11 \\
\hline Russia & 1 & 1 & 0 & 1 \\
\hline Italy & 3 & 0 & 10 & 10 \\
\hline Germany & 3 & 1 & 5 & 6 \\
\hline Portugal & 1 & 0 & 1 & 1 \\
\hline Total & 65 & 121 & 148 & 269 \\
\hline
\end{tabular}

HPP hypophosphatasia

aEnrollment dates: January 2015-September 2017 
enroll. Only patients who had a diagnosis of HPP confirmed by low serum ALP activity for age and sex at any time (but must have been before treatment initiation) and/or an $A L P L$ pathogenic variant were included in this analysis. At minimum, patients must have also had data available for each of the following parameters: asfotase alfa treatment status; date of informed consent; date of birth (or age at enrollment in countries that did not permit collection of birthdate); and sex.

\section{Data collection and handling}

At the time of enrollment, baseline clinical data and information related to HPP disease history are collected. Pretreatment data on HPP disease history are also collected for those who initiated treatment with asfotase alfa before being enrolled in the Registry. Investigators review patients' medical records and submit the data to the sponsor using a secure electronic case report form (eCRF). Race and ethnicity were categorized per US National Institutes of Health recommendations.

The Registry aims to obtain data under conditions of routine clinical care in a real world setting; therefore, some patients may have missing values for some variables given that clinical care practices differ throughout the world. For purposes of data completeness, data queries were generated and distributed to sites for them to address.

\section{Statistical methods}

We report baseline characteristics and medical history by age group at enrollment (children: age $<18 \mathrm{y}$; adults: age $\geq 18$ y). All analyses are descriptive. Continuous variables are described using median (min, max) and mean (standard deviation [SD]), as appropriate. Categorical variables are described using frequencies and percentages. Percentages were based on the available data at the time of the analysis, which vary from variable to variable given the observational nature of the HPP Registry.

\section{Results}

\section{Demographics}

A total of 269 patients from 65 sites in 11 countries (Table 1) were enrolled in the HPP Registry from January 2015 through September 2017 and met the criteria for this analysis. Of these, 121 patients (45.0\%) were children (median [min, max] age at enrollment: $4.3[-0.01,17.3] \mathrm{y}$ )

Table 2 Demographics by age at enrollment

\begin{tabular}{|c|c|c|c|}
\hline Characteristic & Children (age < 18 y) $(n=121)$ & Adults (age $\geq 18$ y) $(n=148)$ & Total $(N=269)$ \\
\hline Age at enrollment, ${ }^{a} y$ & $n=121$ & $n=148$ & $n=269$ \\
\hline Mean (SD) & $5.7(4.7)$ & $48.8(15.4)$ & $29.4(24.5)$ \\
\hline Median (min, max) & $4.3(-0.01,17.3)$ & $51.4(18.5,78.9)$ & $26.0(-0.01,78.9)$ \\
\hline Sex, n (\%) & $n=121$ & $n=148$ & $n=269$ \\
\hline Female & $74(61.2)$ & $108(73.0)$ & $182(67.7)$ \\
\hline Race, ${ }^{\mathrm{b}}$ (\%) & $n=111$ & $n=140$ & $n=251$ \\
\hline White & $64(57.7)$ & $126(90.0)$ & $190(75.7)$ \\
\hline Asian & $35(31.5)$ & $3(2.1)$ & $38(15.1)$ \\
\hline American Indian or Alaska Native & 0 & $2(1.4)$ & $2(0.8)$ \\
\hline Native Hawaiian or other Pacific Islander & 0 & $1(0.7)$ & $1(0.4)$ \\
\hline Other/multiple & $5(4.5)$ & $4(2.9)$ & $9(3.6)$ \\
\hline Not reported & $7(6.3)$ & $4(2.9)$ & $11(4.4)$ \\
\hline Ethnicity, $\mathrm{n}(\%)$ & $n=121$ & $n=147$ & $n=268$ \\
\hline Not Hispanic or Latino & $103(85.1)$ & $116(78.9)$ & $219(81.7)$ \\
\hline Hispanic or Latino & $4(3.3)$ & $18(12.2)$ & $22(8.2)$ \\
\hline Not reported & $14(11.6)$ & $13(8.8)$ & $27(10.1)$ \\
\hline Family history of HPP, n (\%) & $n=117$ & $n=135$ & $n=252$ \\
\hline Yes & $50(42.7)$ & $66(48.9)$ & $116(46.0)$ \\
\hline No & $61(52.1)$ & $54(40.0)$ & $115(45.6)$ \\
\hline Not reported & $6(5.1)$ & $15(11.1)$ & $21(8.3)$ \\
\hline Treated with asfotase alfa at enrollment, n (\%) & $n=121$ & $n=148$ & $n=269$ \\
\hline Yes & $45(37.2)$ & 26 (17.6) & 71 (26.4) \\
\hline
\end{tabular}

HPP hypophosphatasia, SD standard deviation

${ }^{a}$ Negative values for age indicate enrollment date occurred during pregnancy

${ }^{\mathrm{b}}$ The race and ethnicity categories used are those recommended by the US National Institutes of Health 
and $148(55.0 \%)$ were adults $(51.4[18.5,78.9]$ y; Table 2). The majority of children and adults were female (61.2 and $73.0 \%$, respectively) and white (57.7 and $90.0 \%$, respectively).

\section{HPP medical history}

A family history of HPP was reported for nearly half of the children (42.7\%) and adults (48.9\%; Table 2). Approximately half of all patients were diagnosed with HPP in childhood (55.2\%), and half were diagnosed in adulthood (44.8\%). In childhood, diagnoses occurred most frequently between age 2 to $<10$ years $(21.9 \%)$ and before age 6 months (20.4\%). Diagnosis during adulthood was most common at age $\geq 50$ years (20.9\%; Fig. 1 ).

The diagnosis of HPP was delayed relative to age of reported onset of signs or symptoms of the disease in both children and adults (Fig. 2). Children had a median age at earliest reported manifestation of HPP of 7.2 months (min: -2.3 mo, max: $16.0 \mathrm{y}$ ), more than 12 months before median age at HPP diagnosis of 20.4 months (min: -0.2 mo, max: 16.0 y; Fig. 2 a). Median diagnostic delay was $<1$ week $(0.1 \mathrm{mo}$; min: 0 , max: 11.5 y) for children who had first reported manifestation of HPP at age $<1$ year $(n=46)$ and 8.4 months (min: 0, max: 10.7 y) for children with earliest reported HPP manifestation at age $>1$ year $(n=34$; Fig. 2 b). In adults, median age of earliest reported manifestations of HPP was 37.6 years (min: $0.2 \mathrm{y}$, max: $75.2 \mathrm{y}$ ), which preceded median age at diagnosis $(47.5 \mathrm{y} ; \min : 0.2 \mathrm{y}$, max: 75.2 y) by $\sim 10$ years (Fig. 2 c). Median diagnostic delay was 24.5 years (min: 0, max: 46.3 y) for adults who had earliest reported manifestations of HPP before age 18 years $(n=18)$ and 3.8 years (min: 0, max: 21.7 y) for those with first reported manifestation at age $\geq 18$ years $(n=27$; Fig. 2 d).

Premature loss of deciduous teeth $(48.2 \%$ of children aged $\geq 6 \mathrm{mo}$ ), bone deformity (32.5\%), and failure to thrive $(26.7 \%)$ were most commonly reported in the HPP-related disease history of children (Table 3). Pain (74.5\%), orthopedic procedures and therapies (44.6\%), and recurrent and poorly healing fractures (36.5\%) were most commonly reported in the HPP-related disease history of adults (Table 3).

\section{Medication history}

Treatments affecting bone mineralization that were reported in baseline medical histories are summarized in Fig. 3. The majority of children (20/35 [57.1\%]) and adults (47/74 [63.5\%]) for whom data were available had received vitamin $\mathrm{D}$ supplementation. Bisphosphonate use was reported for 2/35 (5.7\%) children and for 13/74 (17.6\%) adults. Of the total number of medications reported, 14/128 (10.9\%) taken by children and $93 / 143(65.0 \%)$ taken by adults were for pain management and $1 / 128(0.8 \%)$ taken by children and 35/143 (24.5\%) taken by adults were antidepressant or anxiolytic medications. Most patients (73.6\%) were not receiving asfotase alfa at enrollment (Table 2).

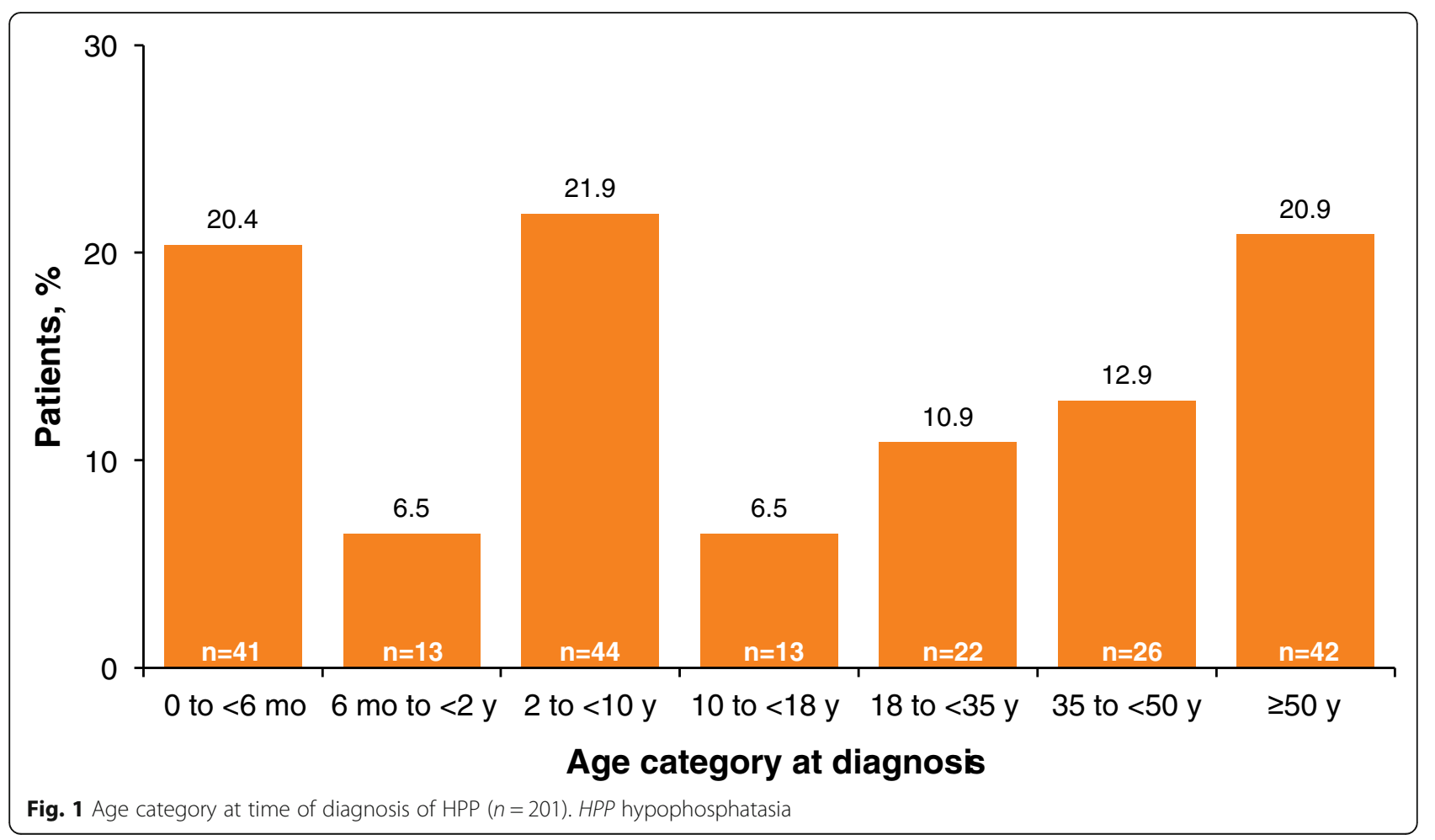


A

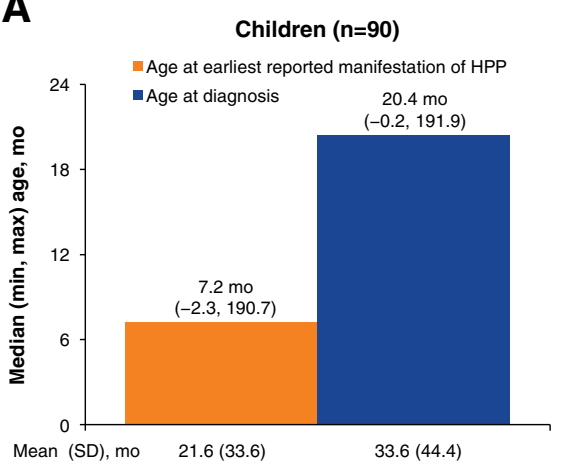

C

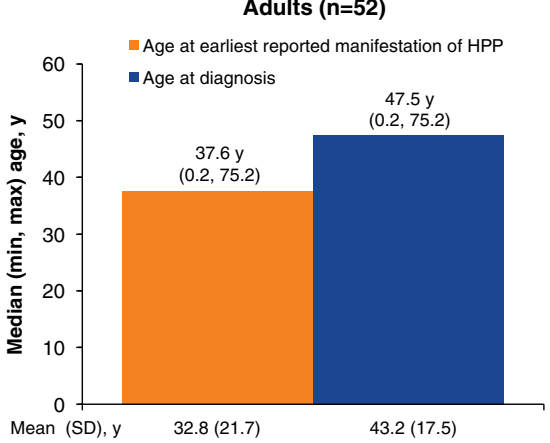

B

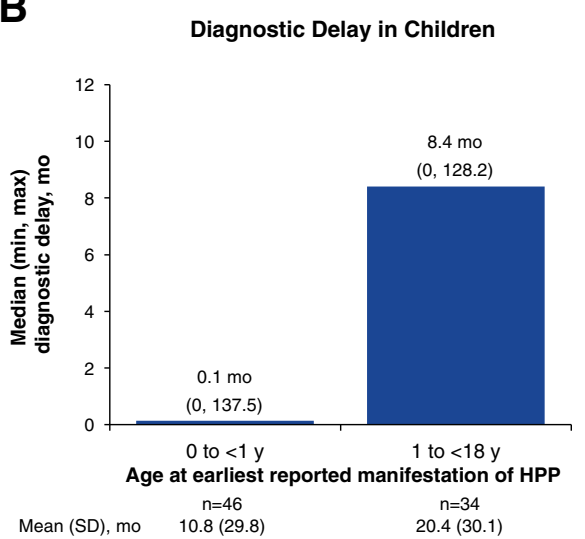

D

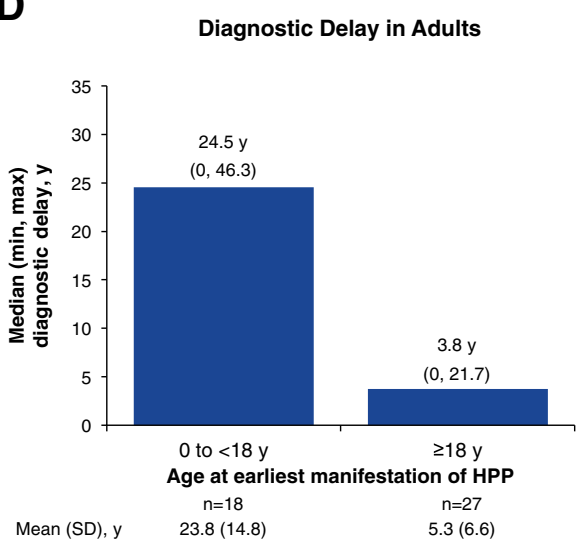

Fig. 2 Age at earliest reported manifestation vs. age at diagnosis of HPP (a: children; c: adults) and diagnostic delay by age at earliest reported manifestation of HPP (b: children; d: adults). Patients with reported age at first reported manifestation occurring after their age at diagnosis were excluded from the analysis of diagnostic delay. Negative values for age indicate a date that occurred during pregnancy. HPP hypophosphatasia

\section{ALPL pathogenic variants}

In total, $172(68.8 \%)$ of 250 patients with submitted data had ALPL pathogenic variant analysis performed. Among the 172 patients (86 children, 86 adults), 218 pathogenic variants (126 in children, 92 in adults) were reported. Missense variants were the most common of all the reported pathogenic variants in children (72.2\%) and adults (76.1\%) (Fig. 4).

\section{Discussion}

The Global HPP Registry represents, to date, the largest real world dataset from patients with HPP. Here we report baseline characteristics and medical history for up to 269 patients from 11 countries who were enrolled in the Registry during its first 2.75 years of recruitment.

Medical histories showed substantial delays between age at earliest reported manifestations and age at diagnosis of HPP in both children and adults. There may be several reasons for this, including low awareness of HPP, heterogeneity of the manifestations of HPP, and lack of specific or routine testing for HPP. Additionally, as laboratories use different methods for assessment of ALP activity, reference ranges often vary, and it is possible that age- and sex-adjusted ALP reference intervals may not have been used or low values may not have been flagged. The diagnostic delay in the Registry population is concerning, given that nearly half of the patients had a family history of HPP. The index of suspicion for HPP should be high in patients with a positive family history to facilitate a timely diagnosis [3], and our findings suggest that family history is not appropriately considered in the diagnosis.

Diagnostic delays were shorter for children with HPP than for adults, possibly reflecting more obvious clinical manifestations coupled with increased awareness of disease manifestations in recent years, and a more thorough clinical and laboratory evaluation. Prolonged diagnostic delays have been reported previously in adults with HPP $[19,20]$. One study of 22 adults with HPP reported a 5-year delay between first signs/symptoms and diagnosis, with the first manifestations of HPP signs/ 
Table 3 HPP-related disease history

\begin{tabular}{|c|c|c|}
\hline \multirow[b]{2}{*}{ Categorya: Symptom/sign } & \multicolumn{2}{|l|}{$\mathrm{n} / \mathrm{N}(\%)$} \\
\hline & $\begin{array}{l}\text { Children } \\
(n=121)\end{array}$ & $\begin{array}{l}\text { Adults } \\
(n=148)\end{array}$ \\
\hline Rheumatic & $25 / 119(21.0)$ & 115/137 (83.8) \\
\hline Calcific periarthritis & 0 & $9(6.6)$ \\
\hline Chondrocalcinosis & 0 & $6(4.4)$ \\
\hline Fibromyalgia & $2(1.7)$ & $13(9.5)$ \\
\hline Pain $^{\mathrm{b}}$ & $23(19.3)$ & $102(74.5)$ \\
\hline Pseudogout & $2(1.7)$ & $8(5.8)$ \\
\hline Skeletal & $53 / 120(44.2)$ & $66 / 137(48.2)$ \\
\hline Bone deformity & $39(32.5)$ & $18(13.1)$ \\
\hline Pseudofractures & $1(0.8)$ & $9(6.6)$ \\
\hline $\begin{array}{l}\text { Recurrent and poorly healing } \\
\text { fractures }\end{array}$ & $5(4.2)$ & $50(36.5)$ \\
\hline Rickets-like changes (by radiograph) & $22(18.3)$ & $8(5.8)$ \\
\hline Orthopedic procedures and therapies & $26 / 120(21.7)$ & $62 / 139(44.6)$ \\
\hline Muscular & 23/119 (19.3) & $51 / 137(37.2)$ \\
\hline Abnormal gait & $17 / 91^{c}(18.7)$ & 28/137 (20.4) \\
\hline Weakness & 15/119 (12.6) & $42 / 137(30.7)$ \\
\hline Premature loss of deciduous teeth & $53 / 110^{d}(48.2)$ & $34 / 137(24.8)$ \\
\hline Renal/metabolic & 28/119 (23.5) & 22/137 (16.1) \\
\hline Hypercalcemia & $15(12.6)$ & $6(4.4)$ \\
\hline Hyperphosphatemia & $7(5.9)$ & $6(4.4)$ \\
\hline Kidney stones & $2(1.7)$ & $7(5.1)$ \\
\hline Nephrocalcinosis & $11(9.2)$ & $5(3.6)$ \\
\hline Neurologic & $32 / 120(26.7)$ & $16 / 137(11.7)$ \\
\hline Craniosynostosis & $12(10.0)$ & $4(2.9)$ \\
\hline Developmental delay & $16(13.3)$ & $3(2.2)$ \\
\hline Increased intracranial pressure & $2(1.7)$ & $1(0.7)$ \\
\hline Seizures & $11(9.2)$ & $12(8.8)$ \\
\hline Failure to thrive & $32 / 120(26.7)$ & $7 / 137(5.1)$ \\
\hline Respiratory support & 22/118 (18.6) & $6 / 138(4.3)$ \\
\hline Invasive ventilation & $18(15.3)$ & 0 \\
\hline CPAP/BiPAP & $7(5.9)$ & $5(3.6)$ \\
\hline Supplemental oxygen & $11(9.3)$ & $2(1.4)$ \\
\hline
\end{tabular}

CPAP continuous positive airway pressure, BiPAP bilevel positive airway pressure, HPP hypophosphatasia

a Patients may have had $>1$ sign/symptom within each category

${ }^{\mathrm{b}}$ Combines generalized body pain, chronic bone pain, and chronic muscle pain ${ }^{c}$ Excludes patients aged $<2$ years at enrollment

${ }^{d}$ Excludes patients aged $<6$ months at enrollment

symptoms occurring at a median age of 44 years and diagnosis at a median age of 49 years [19]. A retrospective case review in 9 adults with HPP reported a median diagnostic delay of 46 years since presentation of dental signs of HPP and 27 years since the first fracture or major adult tooth problems [20]. Further, in our Registry study population, certain age groups were diagnosed with HPP more often, with peaks in diagnoses for those aged $<6$ months, aged 2 to $<10$ years, and aged $\geq 50$ years. This is an interesting observation that may reflect disease severity and/or burden at these ages. Many patients diagnosed with HPP as adults had manifestations in childhood, highlighting the importance of taking thorough medical histories to ensure timely diagnosis.

Baseline medical history from the Registry documented systemic manifestations of HPP across all ages, which were generally consistent with manifestations described in the literature on childhood and adult HPP [19, 21, 22]. Interestingly, skeletal manifestations were present in $<50 \%$ of children (44.2\%) and adults (48.2\%), and specifically, rickets-like changes on radiographs were reported in only $18.3 \%$ of children, highlighting the importance of considering nonskeletal manifestations as part of the diagnosis of HPP. Further, recurrent and poorly healing fractures were less common in children (4.2\%) than adults (36.5\%); however, the data do not reflect whether patients had a history of $\geq 1$ fracture, thereby probably underestimating the overall occurrence of fractures, recurrent fractures, and/or poorly healing fractures.

The need for respiratory support was reported more frequently for children (18.6\%) than adults $(4.3 \%)$. This may be expected because patients with perinatal/infantile HPP often experience respiratory complications, consistent with the natural history of HPP in young children. These data may also be underestimated because patients who were deceased, likely because of respiratory compromise, were not included in the Registry. It is unknown whether the respiratory support reported in the small percentage of adults was provided for symptoms occurring during early childhood or whether this represents respiratory comorbidities.

Pain was commonly reported in the medical histories of patients with HPP (about one fifth of children and three quarters of adults), with approximately 3 times the proportion of adults as children receiving pain medications. The lower rate in children may be an underrepresentation, as young children may be less likely or unable to report pain; it has been reported that parents and medical practitioners often underestimate pain severity in children [23-26]. Our finding in adults is consistent with results of a previous study $(N=125)$, which reported that $95 \%$ of adults with HPP had a history of pain and recent pain [22]. Chronic pain contributes to impairments in physical function, ability to perform activities of daily living, and quality of life in patients with HPP [20, 22], as has been shown for other bone diseases (e.g., osteogenesis imperfecta) [27-29].

The Registry also showed that bisphosphonates are being prescribed to patients with HPP (likely before a diagnosis of HPP). Bisphosphonates should be avoided in patients with HPP because of their biochemical similarity to PPi and because they limit bone turnover 


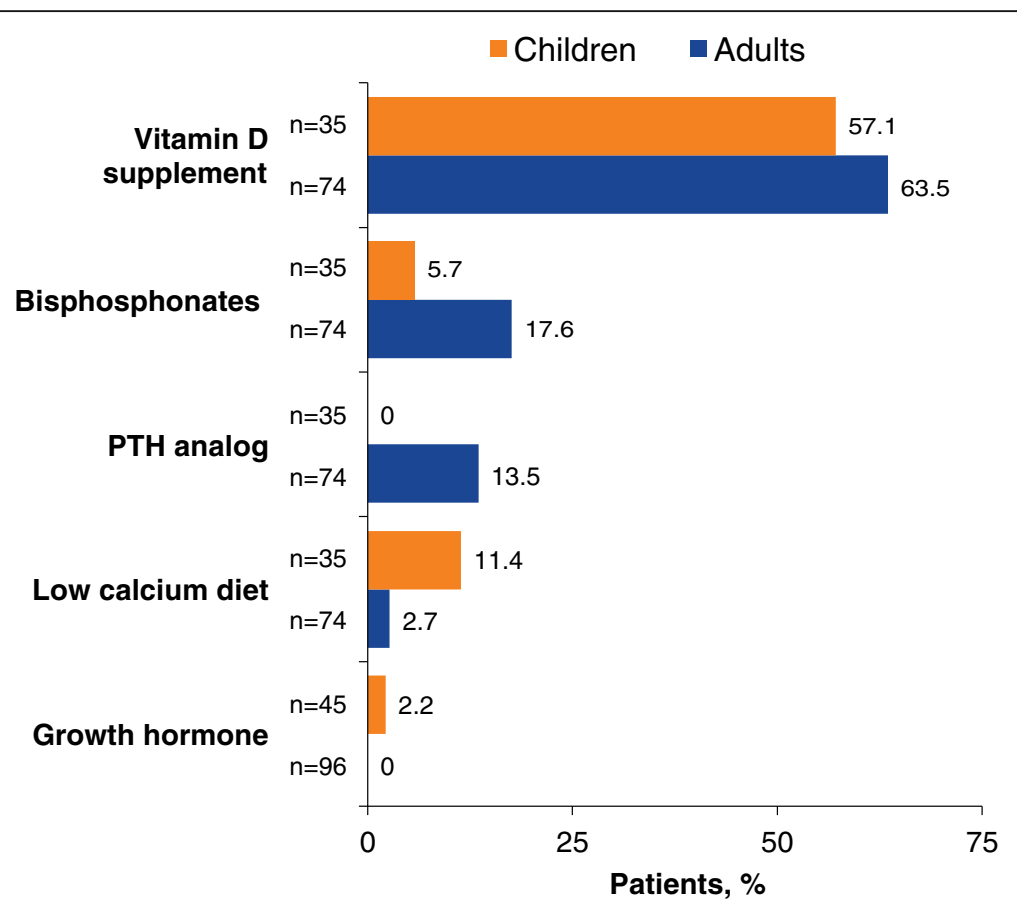

Fig. 3 Treatments affecting bone mineralization reported in medical histories for children and adults enrolled in the HPP Registry. HPP hypophosphatasia; PTH parathyroid hormone

leading to reduced activity of bone-specific ALP [3032]. Case studies of adults with previously undiagnosed HPP have reported increased incidences of atypical femoral fractures during treatment with bisphosphonates [33-36].
As with any registry collecting real world data, there are inherent challenges and limitations, such as ensuring complete data entry [37]. Access to and completeness of patient medical records vary across countries because of differences in medical chart documentation practices,

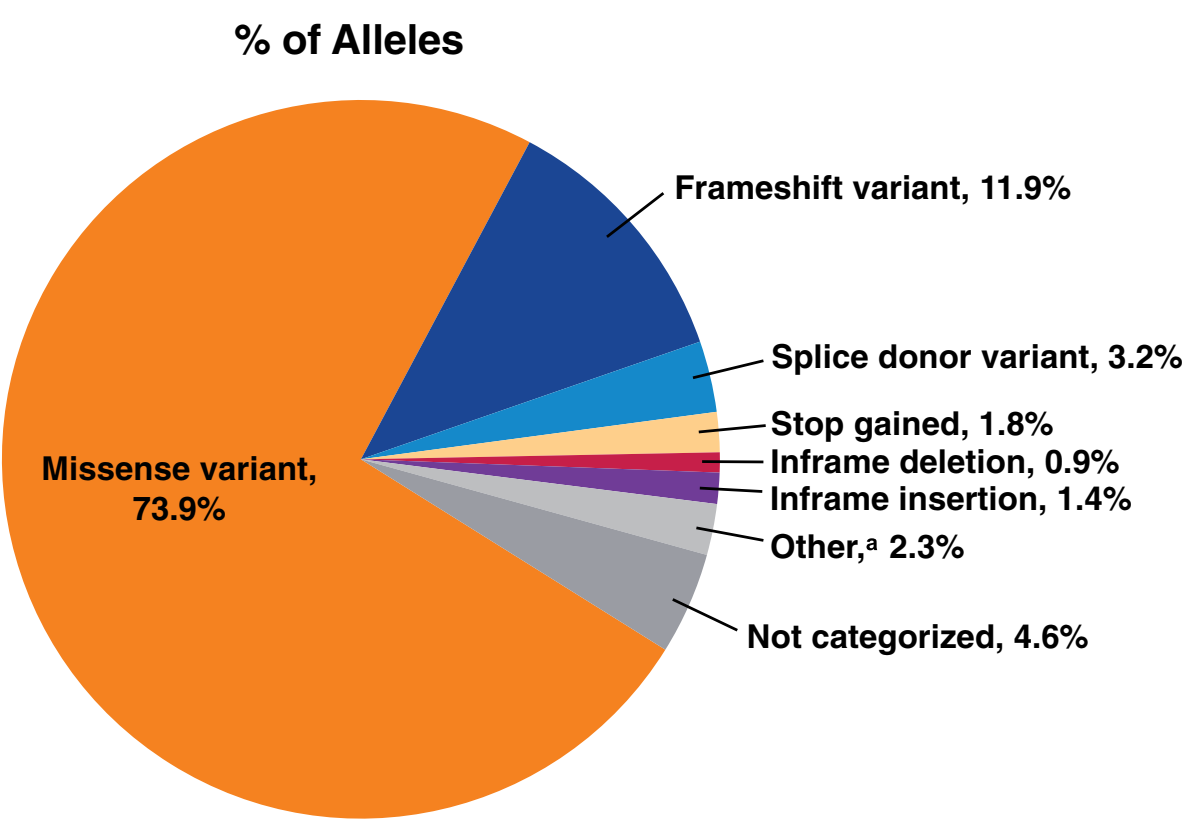

Fig. 4 Frequency of ALPL pathogenic variants. Pathogenic variants were categorized using the definitions from Sequence Ontology [40]. Figure reports the percentages of the total number of pathogenic variants reported $(n=218)$. ${ }^{a}$ Other includes structural variant $(n=2)$, synonymous variant $(n=2)$, and missense variant/splice region variant $(n=1)$ 
limits on time clinicians have to complete data entry, data confidentiality policies, and data linkages within one or more healthcare systems. To address this, missing data were retrospectively collected. Further, data collected for the Registry may have been subject to recall bias, both by the patient and the clinician [37]. In addition, because the Registry did not capture data from patients who were deceased before enrollment, the data may underestimate the full spectrum of disease burden in neonates and young children. Lastly, details on the genotypes of patients in the Registry are not presented, as this information was not available at the time of data analysis [37].

\section{Conclusions}

Baseline data from the HPP Registry provide evidence that delays in the recognition and diagnosis of HPP are common and that HPP is associated with systemic manifestations across all ages. These findings highlight the ongoing need to take thorough medical and family histories and the importance of recognizing the systemic manifestations of HPP to ensure a timely diagnosis. The inclusion of HPP on newborn screening panels should be considered to minimize diagnostic delays and ensure appropriate patient follow-up and management, as has been observed with other genetic diseases [38, 39]. The Global HPP Registry will further improve our understanding of the natural history of HPP and provide longitudinal data on disease burden of HPP, including systemic manifestations, use of concomitant medications that manage manifestations and/or complications of HPP, and potential impact on physical function and quality of life. An improved understanding will allow us to better explore potential geographic, ethnic, and genetic differences in disease manifestations and assess the impact of the approved treatment, asfotase alfa.

\section{Abbreviations}

ALP: Alkaline phosphatase; BiPAP: Bilevel positive airway pressure; CPAP: Continuous positive airway pressure; eCRF: Electronic case report form; HPP: Hypophosphatasia; PLP: Pyridoxal 5'-phosphate; PPi: Inorganic pyrophosphate; PTH: Parathyroid hormone; SD: Standard deviation; TNSALP: Tissue-nonspecific alkaline phosphatase

\section{Acknowledgments}

We wish to thank the patients and their families, investigators, and staff from all sites participating in the HPP Registry. Writing and editorial assistance in the preparation of this manuscript was provided by Lela Creutz, PhD, and Bina J. Patel, PharmD, of Peloton Advantage, LLC, and funded by Alexion Pharmaceuticals, Inc. Mary Kunjappu, PhD, and Mina Patel, PhD, of Alexion Pharmaceuticals, Inc., provided editorial support and critical review of the manuscript.

\section{Funding}

This study was sponsored by Alexion Pharmaceuticals, Inc. (Boston, MA, USA). The Registry is sponsored by Alexion Pharmaceuticals, Inc., and is overseen by a scientific advisory board comprising HPP clinical experts, including employees of Alexion Pharmaceuticals, Inc. Alexion held roles in conception and design of the Registry, collection of data, and statistical analyses, as described in the Authors' Contribution section.

\section{Availability of data and materials}

Qualified academic investigators may request participant-level, de-identified clinical data and supporting documents (statistical analysis plan and protocol) pertaining to this study. Further details regarding data availability, instructions for requesting information, and our data disclosure policy will be available on the Alexion.com website (http://alexion.com/research-development).

\section{Ethics committees}

The protocol was approved by institutional review boards at all sites: Australia: Sydney Children's Hospitals Network Human Research Ethics Committee, Sydney.

Canada: University of Manitoba Biomedical Research Ethics Board, Winnipeg, Manitoba.

France: Advisory Committee for Information Processing in Health Research, Ministry of National Education, Paris.

Germany: Ethics Committee, Institute for Pharmacology and Toxicology, Würzburg.

Italy: Greater Central Area Ethics Committee, Careggi University Hospital,

Florence.

Japan: Only a data transfer agreement was required as this is a

noninterventional study.

Portugal: Health Ethics Committee of Centro Hospitalar de Lisboa Ocidental, Lisbon.

Russia: Independent Interdisciplinary Committee for the Ethical Review of Clinical Trials, Moscow.

Spain: Ethics Committee for Research with Medicines of the University Children's Hospital Niño Jesús of Madrid, Madrid.

UK: West Midlands-Coventry \& Warwickshire Research Ethics Committee, Health Research Authority, Nottingham.

USA: Copernicus Group IRB, Durham, NC.

\section{Authors' contributions}

Study design: WH, CL, HG, SF, AL, KO, AP, CRG, LS, PSK. Study investigator: $\mathrm{WH}, \mathrm{CL}, \mathrm{AL}, \mathrm{KO}, \mathrm{CRG}, \mathrm{LS}, \mathrm{PSK}$. Enrolled patients: $\mathrm{WH}, \mathrm{CL}, \mathrm{AL}, \mathrm{KO}, \mathrm{CRG}, \mathrm{LS}, \mathrm{PSK}$. Collection and assembly of data: $\mathrm{WH}, \mathrm{CL}, \mathrm{AL}, \mathrm{KO}, \mathrm{CRG}, \mathrm{LS}, \mathrm{PSK}$. Data analysis: SF. Data interpretation: WH, CL, HG, SF, AL, KO, AP, CRG, LS, PSK. Manuscript review and revisions: $\mathrm{WH}, \mathrm{CL}, \mathrm{HG}, \mathrm{SF}, \mathrm{AL}, \mathrm{KO}, \mathrm{AP}, \mathrm{CRG}, \mathrm{LS}, \mathrm{PSK}$. Final approval of manuscript: $W H, C L, H G, S F, A L, K O, A P, C R G$, LS, PSK.

\section{Authors' information}

Not applicable.

\section{Ethics approval and consent to participate}

The study protocol was approved by the institutional review board (or local equivalent) of participating study sites and is being conducted in accordance with International Conference on Harmonisation Good Clinical Practice Guidelines and the Declaration of Helsinki. All patients and/or their parent/legal guardians provided written informed consent and approval to release medical records before participation.

\section{Consent for publication}

Not applicable.

\section{Competing interests}

All authors are members of the Scientific Advisory Board responsible for oversight of the HPP Registry.

$\mathrm{CL}, \mathrm{WH}, \mathrm{AL}, \mathrm{KO}$, and $\mathrm{CRG}$ are clinical study investigators and have received consultancy fees, and/or institutional research funding, and/or grant support, and/or travel support from Alexion Pharmaceuticals, Inc. At the time of the study, WH was affiliated with University of Birmingham, Birmingham, UK. LS is a clinical study investigator and has received consultancy fees and research grants to his institution from Alexion Pharmaceuticals, Inc. SF and AP are employees of and may own stock/options in Alexion Pharmaceuticals, Inc., which sponsored the study. HG was an employee of and may own stock/options in Alexion Pharmaceuticals, Inc., which sponsored the study. PSK is a clinical study investigator and has received consultancy fees and travel support from Alexion Pharmaceuticals, Inc. for consulting and participation on advisory boards. 


\section{Publisher's Note}

Springer Nature remains neutral with regard to jurisdictional claims in published maps and institutional affiliations.

\section{Author details}

'Department of Pediatrics and Adolescent Medicine, Johannes Kepler University Linz, Linz, Austria. ${ }^{2}$ Feinberg School of Medicine, Northwestern University and Lurie Children's Hospital, Chicago, IL, USA. ${ }^{3}$ Alexion Pharmaceuticals, Inc., Boston, MA, USA. ${ }^{4}$ APHP, Bicêtre Paris-Sud, University Paris Sud, Paris-Saclay, Le Kremlin Bicêtre, Paris, France. ${ }^{5}$ Department of Pediatrics, Osaka University, Graduate School of Medicine, Suita, Osaka, Japan. ${ }^{6}$ Rady Faculty of Health Sciences, Max Rady College of Medicine, and Children's Hospital Research Institute of Manitoba, University of Manitoba Winnipeg, Manitoba, Canada. 'Orthopaedic Clinic, König-Ludwig-Haus, University of Würzburg, Würzburg, Germany. ${ }^{8}$ Department of Pediatrics, Duke University Medical Center, 2301 Erwin Rd, Durham, NC 27710, USA.

Received: 14 June 2018 Accepted: 16 January 2019

Published online: 14 February 2019

\section{References}

1. Weiss MJ, Cole DE, Ray K, Whyte MP, Lafferty MA, Mulivor RA, et al. A missense mutation in the human liver/bone/kidney alkaline phosphatase gene causing a lethal form of hypophosphatasia. Proc Natl Acad Sci U S A. 1988;85(20):7666-9.

2. Whyte MP. Hypophosphatasia and how alkaline phosphatase promotes mineralization. In: Thakker RV, Whyte MP, Eisman J, Igarashi T, editors. Genetics of bone biology and skeletal disease. 2nd ed. San Diego, CA: Elsevier (Academic Press); 2018. p. 481-504.

3. Rockman-Greenberg C. Hypophosphatasia. Pediatr Endocrinol Rev. 2013; 10(Suppl 2):380-8.

4. Taillandier A, Domingues C, Dufour A, Debiais F, Guggenbuhl P, Roux C, et al. Genetic analysis of adults heterozygous for ALPL mutations. J Bone Miner Metab. 2018;36(6):723-33.

5. Russell RG. Excretion of inorganic pyrophosphate in hypophosphatasia. Lancet. 1965;2(7410):461-4.

6. Whyte MP, Mahuren JD, Vrabel LA, Coburn SP. Markedly increased circulating pyridoxal-5'-phosphate levels in hypophosphatasia. Alkaline phosphatase acts in vitamin B6 metabolism. J Clin Invest. 1985;76(2):752-6.

7. Whyte MP. Physiological role of alkaline phosphatase explored in hypophosphatasia. Ann N Y Acad Sci. 2010;1192:190-200.

8. Fraser D. Hypophosphatasia. Am J Med. 1957;22(5):730-46.

9. Mornet E, Yvard A, Taillandier A, Fauvert D, Simon-Bouy B. A molecularbased estimation of the prevalence of hypophosphatasia in the European population. Ann Hum Genet. 2011;75(3):439-45.

10. Greenberg CR, Evans JA, McKendry-Smith S, Redekopp S, Haworth JC, Mulivor R, et al. Infantile hypophosphatasia: localization within chromosome region 1p36.1-34 and prenatal diagnosis using linked DNA markers. Am J Hum Genet. 1990;46(2):286-92.

11. Taketani T, Onigata K, Kobayashi H, Mushimoto Y, Fukuda S, Yamaguchi S. Clinical and genetic aspects of hypophosphatasia in Japanese patients. Arch Dis Child. 2014;99(3):211-5.

12. Watanabe A, Karasugi T, Sawai H, Naing BT, Ikegawa S, Orimo H, et al. Prevalence of c.1559delT in ALPL, a common mutation resulting in the perinatal (lethal) form of hypophosphatasia in Japanese and effects of the mutation on heterozygous carriers. J Hum Genet. 2011;56(2):166-8.

13. Hofmann C, Girschick H, Mornet E, Schneider D, Jakob F, Mentrup B. Unexpected high intrafamilial phenotypic variability observed in hypophosphatasia. Eur J Hum Genet. 2014;22(10):1160-4.

14. Hofmann C, Girschick HJ, Mentrup B, Graser S, Seefried L, Liese J, et al. Clinical aspects of hypophosphatasia: an update. Clin Rev Bone Miner Metab. 2013;11:60-70.

15. Bishop N. Clinical management of hypophosphatasia. Clin Cases Miner Bone Metab. 2015;12(2):170-3.

16. Strensia [package insert]. Boston, MA: Alexion Pharmaceuticals, Inc; January 2018.

17. Strensiq [summary of product characteristics]. Rueil-Malmaison, France: Alexion Europe; May 14, 2018.

18. Alexion Pharmaceuticals, Inc.: Strensiq (asfotase alfa) receives marketing approval in Japan for treatment of patients with hypophosphatasia (HPP) [press release]: 6 July 2015. http://www.businesswire.com/news/home/
20150706005254/en/Strensiq\%C2\%AE-asfotase-alfa-Receives-MarketingApproval-Japan. Accessed: 12 June 2018.

19. Berkseth KE, Tebben PJ, Drake MT, Hefferan TE, Jewison DE, Wermers RA. Clinical spectrum of hypophosphatasia diagnosed in adults. Bone. 2013;54(1):21-7.

20. Mori M, DeArmey SL, Weber TJ, Kishnani PS. Case series: odontohypophosphatasia or missed diagnosis of childhood/adult-onset hypophosphatasia? - call for a long-term follow-up of premature loss of primary teeth. Bone Rep. 2016;5:228-32.

21. Whyte MP, Madson KL, Phillips D, Reeves A, McAlister WH, Yakimoski A, et al. Asfotase alfa therapy for children with hypophosphatasia. JCI Insight. 2016;1(9):e85971.

22. Weber TJ, Sawyer EK, Moseley S, Odrljin T, Kishnani PS. Burden of disease in adult patients with hypophosphatasia: results from two patient-reported surveys. Metabolism. 2016;65:1522-30.

23. Brudvik C, Moutte SD, Baste V, Morken T. A comparison of pain assessment by physicians, parents and children in an outpatient setting. Emerg Med J. 2017;34(3):138-44.

24. Singer AJ, Gulla J, Thode HC, Jr. Parents and practitioners are poor judges of young children's pain severity. Acad Emerg Med 2002;9(6):609-612.

25. Khin Hla T, Hegarty M, Russell P, Drake-Brockman TF, Ramgolam A, von Ungern-Sternberg BS. Perception of pediatric pain: a comparison of postoperative pain assessments between child, parent, nurse, and independent observer. Paediatr Anaesth. 2014;24(11):1127-31.

26. Rajasagaram U, Taylor DM, Braitberg G, Pearsell JP, Capp BA. Paediatric pain assessment: differences between triage nurse, child and parent. J Paediatr Child Health. 2009:45(4):199-203.

27. Balkefors V, Mattsson E, Pernow Y, Saaf M. Functioning and quality of life in adults with mild-to-moderate osteogenesis imperfecta. Physiother Res Int. 2013;18(4):203-11.

28. Dahan-Oliel N, Oliel S, Tsimicalis A, Montpetit K, Rauch F, Dogba MJ. Quality of life in osteogenesis imperfecta: a mixed-methods systematic review. Am J Med Genet A. 2016;170a(1):62-76.

29. Hill CL, Baird WO, Walters SJ. Quality of life in children and adolescents with osteogenesis imperfecta: a qualitative interview based study. Health Qual Life Outcomes. 2014:12:54

30. Whyte MP. Hypophosphatasia - aetiology, nosology, pathogenesis, diagnosis and treatment. Nat Rev Endocrinol. 2016:12(4):233-46.

31. Kishnani PS, Rush ET, Arundel P, Bishop N, Dahir K, Fraser W, et al. Monitoring guidance for patients with hypophosphatasia treated with asfotase alfa. Mol Genet Metab. 2017;122(1-2):4-17.

32. Vaisman DN, McCarthy AD, Cortizo AM. Bone-specific alkaline phosphatase activity is inhibited by bisphosphonates: role of divalent cations. Biol Trace Elem Res. 2005;104(2):131-40.

33. Whyte MP. Atypical femoral fractures, bisphosphonates, and adult hypophosphatasia. J Bone Miner Res. 2009;24(6):1132-4.

34. Sutton RA, Mumm S, Coburn SP, Ericson KL, Whyte MP. "Atypical femoral fractures" during bisphosphonate exposure in adult hypophosphatasia. J Bone Miner Res. 2012;27(5):987-94.

35. Cundy T, Michigami T, Tachikawa K, Dray M, Collins JF, Paschalis EP, et al. Reversible deterioration in hypophosphatasia caused by renal failure with bisphosphonate treatment. J Bone Miner Res. 2015;30(9):1726-37.

36. Genest F, Seefried L. Subtrochanteric and diaphyseal femoral fractures in hypophosphatasia--not atypical at all. Osteoporos Int. 2018;29(8):1815-25.

37. Gliklich R, Dreyer N, Leavy M. In: Gliklich RE, Dreyer NA, Leavy MB, editors. Registries for Evaluating Patient Outcomes: A User's Guide. 3rd ed. Rockville, MD: Agency for Healthcare Research and Quality; 2014.

38. Farrell PM, Lai HJ, Li Z, Kosorok MR, Laxova A, Green CG, et al. Evidence on improved outcomes with early diagnosis of cystic fibrosis through neonatal screening: enough is enough! J Pediatr. 2005;147(3 Suppl):S30-6.

39. Streetly A, Sisodia R, Dick M, Latinovic R, Hounsell K, Dormandy E. Evaluation of newborn sickle cell screening programme in England: 2010-2016. Arch Dis Child. 2018;103(7):648-53.

40. The Sequence Ontology [collaborative oncology project]: Gene Ontology Consortium; 2016 [updated 2018]. http://www.sequenceontology.org/. Accessed June 12, 2018. 